\title{
DEVELOPMENT OF TERRESTRIAL LASER SCANNING SIMULATOR
}

\author{
Thomas Luhmann ${ }^{1}$, Maria Chizhova ${ }^{2}$, Denys Gorkovchuk ${ }^{3,4}$, Darius Popovas ${ }^{1,5}$, Julia Gorkovchuk ${ }^{3}$, Mona Hess ${ }^{2}$
}

${ }^{1}$ Jade University of Applied Sciences, Institute for Applied Photogrammetry and Geoinformatics, Oldenburg, Germany (darius.popovas, luhmann)@jade-hs.de

${ }^{2}$ University of Bamberg, Digital Technologies in Heritage Conservation, Centre of Heritage Conservation Studies and Technologies, Germany-(maria.chizhova, mona.hess)@uni-bamberg.de

${ }^{3}$ Kiev National University for Construction and Architecture, Department of Geoinformatics and Photogrammetry Kyiv, Ukraine denys.gorkovchuk@spm3d.com,gorkovchukjulia@gmail.com

${ }^{4}$ SPM3D LLC, Kyiv, Ukraine. - denys.gorkovchuk@spm3d.com

${ }^{5}$ Vilnius Gediminas Technical University, Department of Geodesy and Cadastre, Vilnius, Lithuania - darius.popovas@vilniustech.lt

\section{Commission II}

KEY WORDS: Simulator, laser scanning, point cloud, virtual reality, game engine.

\begin{abstract}
:
Within the project VRscan3D, funded by DAAD, a terrestrial laser scanner simulator has been developed as educational tool for learning and teaching laser scanning processes. The main goal of the VirScan3D project is to cover engineering digitisation and will be solved through the development of a virtual system that allows users to create realistic data in the absence of a real measuring device in a modelled real-life environment (digital twin). The implementation of the virtual laser scanner is realised within a game engine, which allows for fast and easy 3D visualisation and navigation. Real-life buildings and urban surroundings can be digitised, modelled and integrated into the simulator, thus creating a digital copy of a real-world environment. This article describes the technical realization of the simulator and its evaluation as well as usability testing results conducted by independent users from university courses.
\end{abstract}

\section{INTRODUCTION}

The current situation, namely the ongoing Corona crisis, has demonstrated that digitalization of teaching is not only an option, but the indispensable necessity of present reality. The pandemic has particularly affected those academic programs where classroom teaching is unavoidable, for example, in practical courses with surveying equipment. The project VRscan3D is designed to develop a virtual laser scanner simulator and related teaching concepts to support universities and user groups that have no or only very limited access to real terrestrial laser scanning equipment. This project is funded by DAAD (German Academic Exchange Service) within a program for supporting the internationalisation of Ukrainian universities: "GermanUkrainian higher education institution collaborations". The project group consists of two German universities and two Ukrainian universities based on a long-term cooperation that has already started in 2002 .

Terrestrial laser scanners (TLS) are nowadays used extensively in geoinformation, heritage, archaeology, construction, and surveying industries. Applying TLS, complex object geometries (historical buildings, industrial plants, etc.) of almost any shape can be recorded three-dimensionally in a short time, usually from a number of different scan stations. Modern laser scanners also consist of integrated cameras providing photogrammetric image processing, or support of registration processes.

Typical laser scanners today cost between 35,000 and 80,000 Euro per device. This makes it difficult for some educational institutions, especially in less developed countries, to supply such devices for training of students. Moreover, the recent pandemic situation forced to switch to online teaching. Such disciplines as digital heritage technologies, surveying, geomatics etc. require hands on training to use specific equipment, which is rather difficult to implement online. In this paper, we are presenting the latest results of the developed terrestrial laser scanner (TLS) simulator VRscan3D, based on the game programming environment Unreal Engine. The software allows the planning and simulation of practice-oriented surveying procedures with laser scanners within a given virtual $3 \mathrm{D}$ environment. The use of such simulator makes it possible to overcome above-mentioned limitations, without the need for expensive equipment and could be offered online.

A basic version of the software with limited functionality can be downloaded for free. Advanced professional versions will be available in the near future. 


\section{RELATED WORKS}

The use of simulation for e-learning purposes has fully proven its positive impact for education (Falloon, 2019). In summary, simulation of complex technical processes is stated as a valuable tool for e-learning, or at least a significant extension to classical teaching concepts. In the higher education, innovative training methods increasingly include video games that have proven a positive impact on learning goals, balanced with conventional training methods (Vlachopoulus \& Makri, 2017). Especially in geospatial sciences, the simulation in virtual environments is mainly used for the virtual reconstruction of natural scenes like cultural heritage objects or 3D cities and its real time manipulation (Kersten \& Edler, 2020; ISPRS, 2020 WG IV/9 Geovisualization, Augmented and Virtual Reality).

Recently, the simulation of modern geodetic instruments is rather unusual, but some attempts were made to simulate a classical instrument in virtual environment (Dib et al., 2014; Kuo et al., 2011).

The concept and initial results on laser scanner simulator were previously published by (Chizhova et al., 2020) and (Popovas et al., 2021). This report summarizes the basic technological background and presents newest software features and user feedback.

\section{DEVELOPED METHODOLOGY}

Main target groups of VRscan3D simulator are students and teachers of geomatics, heritage, construction and other related higher education departments with limited access to expensive TLS equipment. The program allows to simulate all processes of the fieldwork phase of terrestrial laser scanning and generate results comparable with the data generated by real physical scanners on-site. Moreover, integrated digital environment can simulate real world objects, i.e., buildings or sites. These results can be processed further in common software packages, analogous to real surveying practice (s. Fig. 1).

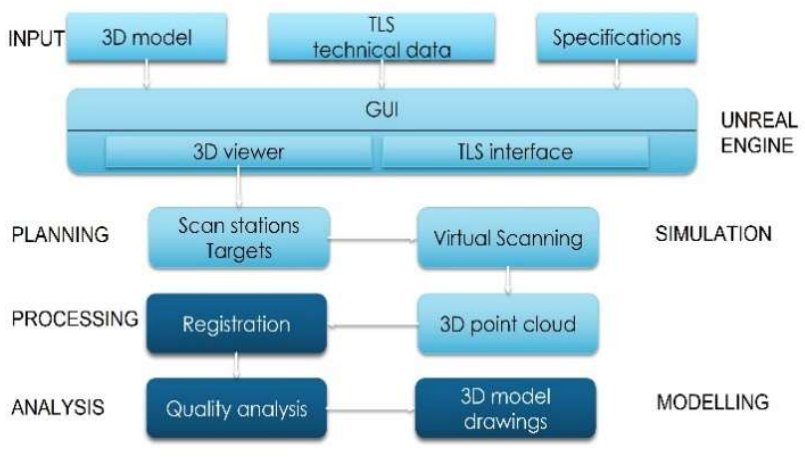

Figure 1. Simulator functionality.

Light blue textboxes in Figure 1 top show the functionality covered by VRscan3D. Dark blue textboxes illustrate further processing steps of the simulated data using user-selected software. Users will be able to select and configure custom scanner parameters like resolution, field of view, min. and max. range, angular accuracy and range accuracy.
During simulation, an angular grid of rays is projected from the scanner centre and the intersection of each ray with the closest surface gives the discrete point coordinates. The maximum range of surface search is limited to the scanner range specification. The result of the simulation is a structured 3D point cloud in different file formats for import in further processing software. For each scan station, a single point cloud is generated given in the local scanner coordinate system.

\section{TECHNICAL DETAILS}

VRscan3D is developed within Unreal Engine, hence user's computers should satisfy its minimum requirements (Unreal, 2020). Main simulator functions are programmed with Blueprint Visual Scripting system of Unreal Engine. That makes the system very flexible and allows modifications of software modules without recompiling the whole code. Some modules were programmed with $\mathrm{C}++$ language due to Blueprint limitations and to improve the overall performance of the software. Virtual environment 3D models (in IFC format) are imported to Unreal engine using Datasmith plugin.

The product functionality includes following features:

- selecting the scanner model which will be simulated;

- laser scanning process simulation to obtain point clouds with intensity and noise values based on scanner specifications;

- scan station management, which allows to specify settings (resolution) for each scan station, create, move and delete stations;

- target management which allows placing and removing targets of different types (checkerboard and spherical);

- project management, which allows to save the project and to continue work later;

- batch scanning, i.e. placing multiple scan stations at the same time and triggering scanning simulation for all stations one by one;

- real-time visualisation of scanning process and generated point clouds;

- exporting the point cloud to standard file formats for further post-processing.

The simulator offers two scenarios of work:

1) The user is navigating within the virtual environment and selects and places target positions as in real life. Then the scanner position and scan settings are specified and the scanning process of the virtual device is started. The progress of point-cloud collection is visualised in real time by a projected scanner beam. When the scanning is finished the user avatar 'goes' to next station.

2) The user navigates within the virtual environment and selects positions for all targets and all scan stations. Subsequently, scan settings are specified for all stations or for each station separately. Finally, the scanning simulation is started for all stations in batch mode.

Currently a basic generic (no name) scanner and three professional scanners are implemented. The list of implemented scanners will be extended continuously. For all scanners relevant scanning parameters can be set. In case of professional models, 
parameter setting is emulated by realistic user interfaces that offer the same look-and-feel impression as for the real devices.

In the recent version of software, noise and intensity simulation is also implemented based on mathematical and physical models. Since simulated material properties are available (specified during modelling) for each part of the 3D scene model, noise and intensity are simulated as a function of material characteristics, scanner specifications and reflection properties (see example in Fig. 5). Laser beam propagation and reflection from glass and other highly reflective surfaces also simulated in the recent version of simulator software (see example in Fig. 5 and Fig.6).

Many terrestrial scanners have integrated cameras for colorizing point clouds. However, camera parameters and orientation are not open for users. Typically, this data is processed internally in native scanner software with coloured point cloud and spherical panoramas as a result. Therefore, it was decided to simulate directly spherical panoramas from scan stations (Figure 2).

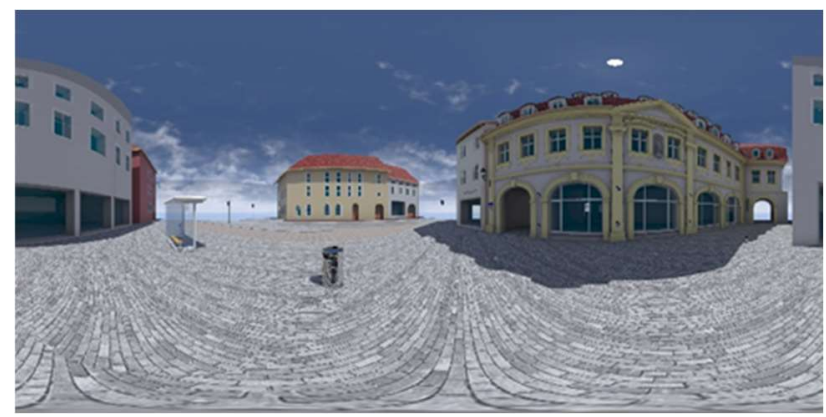

Figure 2. Example of spherical panorama image, generated from simulator.

Unlike real scanning, where the speed depends mostly on physics, the speed of the simulation depends on several factors:

- computing power;

- openness or obstruction/occlusion of the environment;

- presence of surfaces that reflect or refract the laser beam;

- noise simulation.

Computing power has a direct impact on scanning speed. Faster CPU and GPU provide faster simulation. At the same time the simulator doesn't require high amounts of memory, as simulated points are stored in RAM for a short time and then written directly to disk. Memory consumption typically is at $0.5-3 \mathrm{~GB}$, depending on the scan settings and environment.

The simulation is also faster for open environments, as the number of calculations is significantly reduced when the laser beam does not hit any surface.

Presence of reflective surfaces slow down the simulation speed, as there are additional calculations for reflected laser beam and intensity.

Simulation of range and angular noise require very simple calculations, therefore its effect on scanning speed is less than $1 \%$.

For an average PC with Intel 9-11 Gen processor and RTX family graphic card, simulation speed is at level 100-500 thousand points per second. It is approximately at the level of Leica
BLK360 speed (360 thousand points per second), which is significantly slower than modern phase-based scanners with 1-2 million points per second. But it is worth to mention that such maximum speed in real life is typically available for short range scans, and using the maximum available distance requires reducing of scanning speed, whereas speed of simulation is independent of scanning range.

Generation of images is one of the aspects, where simulation is much faster than in reality. For real scanners, image capturing takes 1 minute for systems with several wide-angle cameras (e.g. RTC360) and up to 30 minutes for systems with single camera and narrow field-of-view (e.g. Leica C10). The generation of simulated images takes only 5-7 seconds.

To extend the simulator to real VR environment, a prototype for Oculus Rift S headset was developed (Fig. 3). It uses the computing power of the PC, so the core of the simulator does not need to be changed. However, additional interfaces for interaction within an VR environment need to be developed.

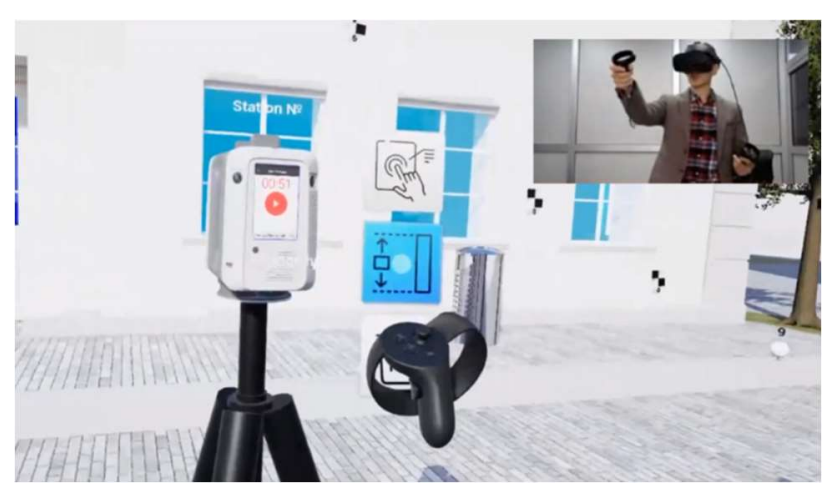

Figure 3. Operating simulator in Oculus Rift S VR headset.

There are two formats for point cloud export supported in the current version: TXT and PTX. Both are text files for easy use in any external software. PTX is able to store transformation parameters for registered point clouds. Since many of modern scanners have additional sensors for coarse on-site registration of point clouds (e.g. Visual Inertial System on RTC360) it is also possible to simulate such pre-registration and provide users with experience close to reality. The implementation of the E57 format as vendor-neutral binary format for storing of point clouds and images from scanner is planned for future versions.

\section{TEACHING CONCEPT}

Over the past few years, virtual reality technologies in the educational process have evolved from potentially promising to highly effective (DiKuLe 2022, SmartDidaktik 2022). Despite the obvious need to implement distance and mixed learning today, the issues of acquiring skills in working with complex technical equipment remain open for engineering education. The professional competences of engineers, surveyors, geoinformatics engineers or cartographers include the knowledge of modern technologies and the ability to use the latest surveying equipment and software for the collection and processing of geospatial data.

Terrestrial laser scanning systems are one of the most common and modern means of performing engineering surveys in various 
fields. The use of a laser scanner simulator in conditions of limited access to high-cost systems and equipment provides a full cycle of work on the collection, processing and modelling of spatial data in a virtual environment, which leads to the training of practical skills.

Maximum approximation to real working conditions without the need for real measurements with the time-consuming process of coordinating access to equipment and facilities, increases the level of training of students both within educational institutions and training programs. Students of related fields, scientists in multidisciplinary research or other specialists interested in mastering the technology of terrestrial laser scanning can gain basic concepts and skills of working in a virtual simulator with the example of real buildings and structures.

Moreover, the simulated datasets (point clouds) are very close to real scanner generated datasets, therefore suitable for teaching of data management, registration, cleaning, modelling, etc. Apart from this it is foreseen to implement elements of serious gaming (e.g. automatic evaluation of completeness of simulated scan), which will make learning more immersive and attractive.

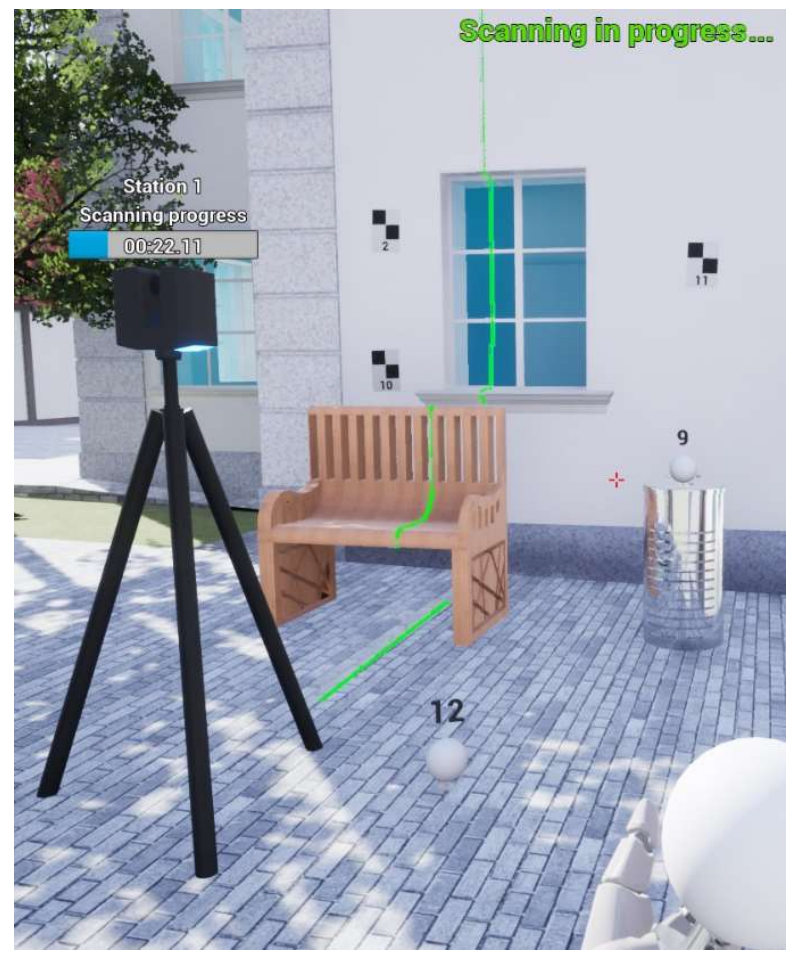

Figure 4. Simulator environment example with scanner and different types of targets.

\section{DEVELOPED METHODOLOGY}

With the VRscan3D simulator developed so far, it is possible to create realistic environmental scenarios that largely meet the requirements from practical projects. It is possible to move interactively with an avatar, define measuring stations and targets, select different scanner types with their respective specifications or use custom settings (Fig. 4).
Figure 4 displays the ongoing simulation where a rotating green line simulates the laser beam. The indicator above the scanner shows the remaining simulation/scan time.

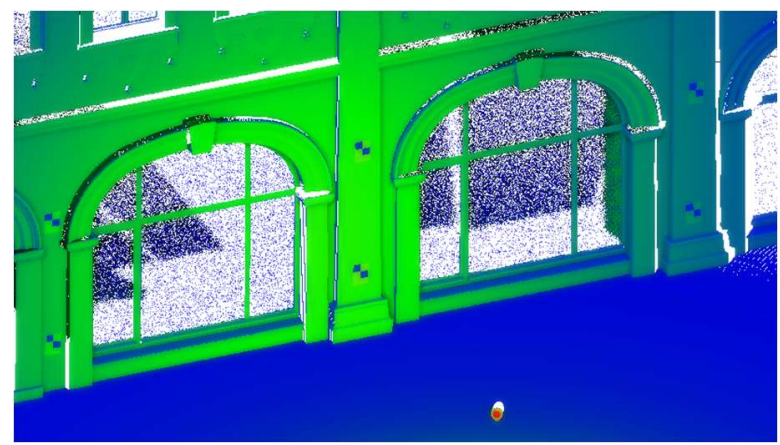

Figure 5. Example of point cloud, generated by the simulator.

Figure 5 represents an example of point cloud, generated by the simulator. The spherical and checkerboard targets are clearly distinguishable in the simulated point cloud, also different intensity values can be observed. Since laser beam propagation through glass is also simulated, some points are visible inside the building, behind the window.

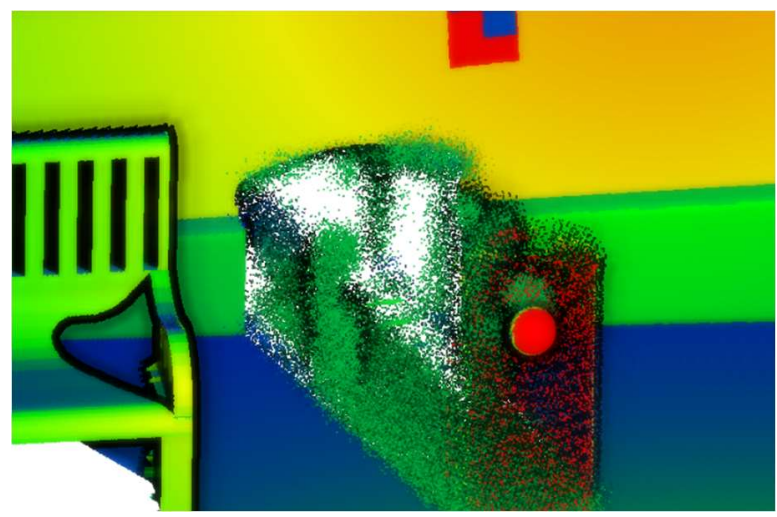

Figure 6. Noise simulation from highly reflective material.

Figure 6 shows noisy data simulated around a highly reflective object (chrome trash bin). The same bin is visible in Figure 4.

During the project duration, two user tests of the developed simulator have been carried out. Both tests were performed as reuseable rated questionnaires with free text option. The first beta version of the simulator has been tested at 2021 by a group of 22 master students at the University of Bamberg, Germany. Their educational backgrounds were quite heterogeneous, ranging from engineering to humanities. Generally, the testing allowed to define the tendencies for further development of the simulator according to the participants' suggestions and identify several errors and bugs (Chizhova et al. 2021).

This year (2022) s smaller group of students from Bamberg University tested the simulator within the 3D object digitisation seminar. All the students had an introduction to laser scanning as part of their master course, but most of them had no experience in real surveying (approx..75\%). Furthermore, many participants were experienced in the computer games (s. Fig. 7). 

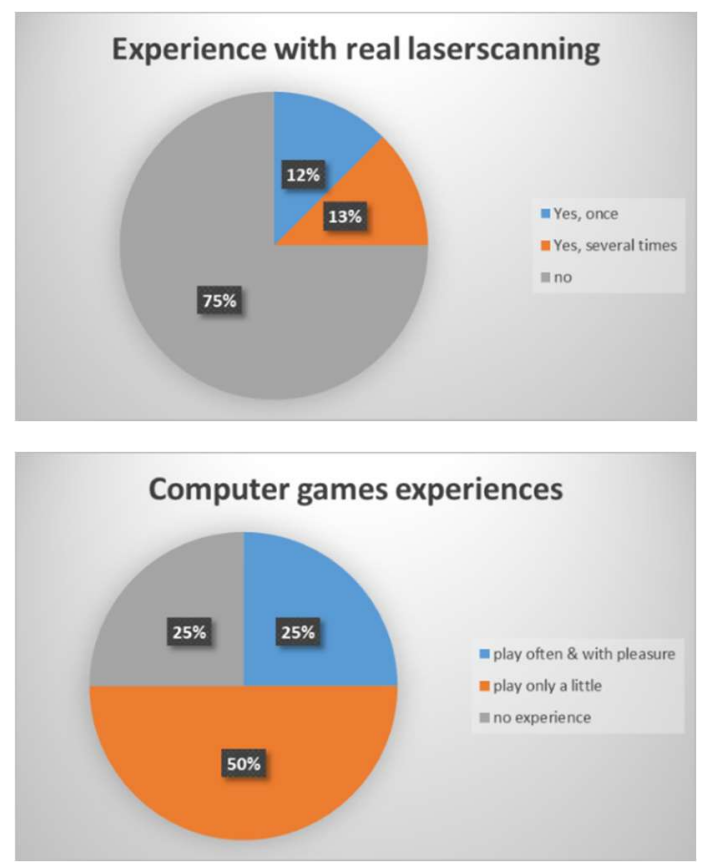

Figure 7. Competencies in laser scanning and games (graphic from the second user testing).

The building shown in the figures was previously scanned with the BLK and FARO laser scanners by the VRscan3D team, then modelled in Autodesk Revit, with the assignment of appropriate building materials, and integrated this into the simulator environment (s. Fig. 8). During the test, participants were asked to use the simulator to virtually scan the historic campus building. Afterwards, the students had the practice with real scanners, capturing the same building.

The participants in the second user testing in both user tests pointed out, that the software runs fluently and provides good insights into laser scanning. It is well suited for trying out and getting to feel the subject matter. Likewise, it allows you to test the positioning of the scanners and targets and easily determine the effects on the result. Most of the functions are very intuitive. The help options are of great use. Furthermore, it could be very applicable for teaching and measurement planning, confirmed by the questionnaire results (s. Fig. 9).

In practice, some issues applied easily in the simulator can be difficult to imagine in reality, like:

- placing targets on any surface (e.g. on the roof),

- jumping or teleporting with equipment in hands,

- the absence of obstacles such as pedestrians or cars;

- etc.

Some of those observations of beta testers might be solved in future versions of simulator software. Randomly passing cars and pedestrians can be integrated, perhaps the user can adjust intensity of the traffic in the options.
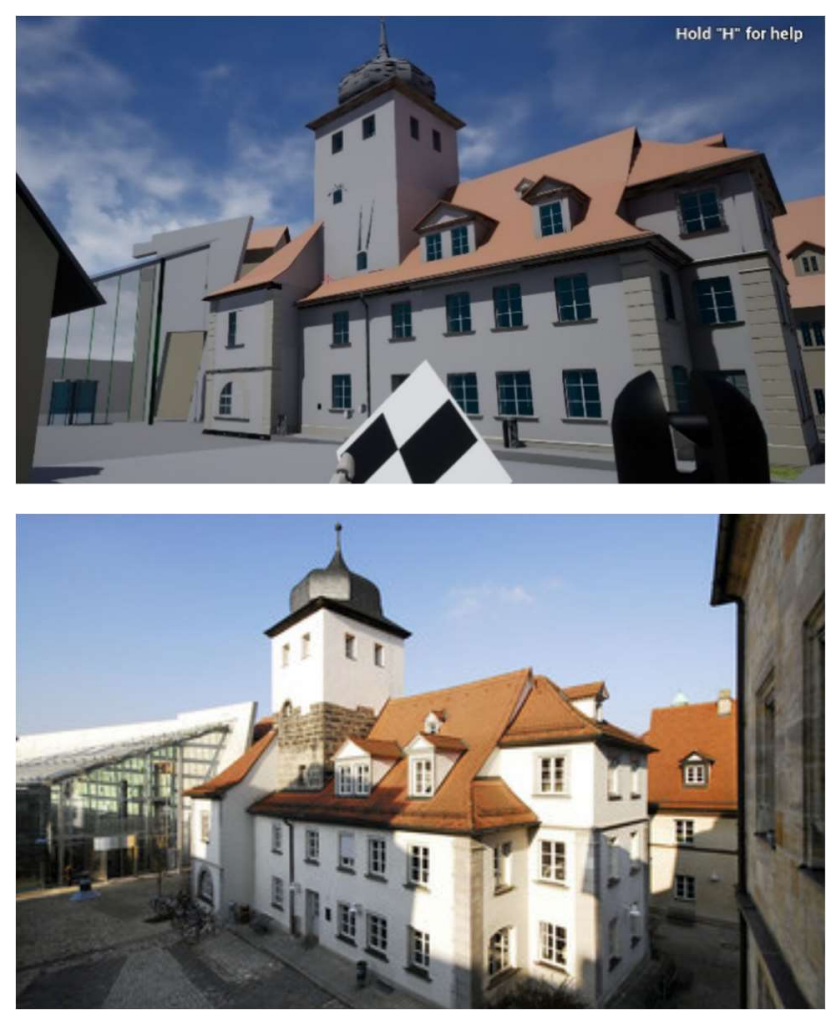

Figure 8. Virtual above and real test object below (University office building 'An der Universität 9', University of Bamberg).

Furthermore, it was recommended by the beta testers to develop a tutorial with an example and workflow for the participants without laser scanning experience.

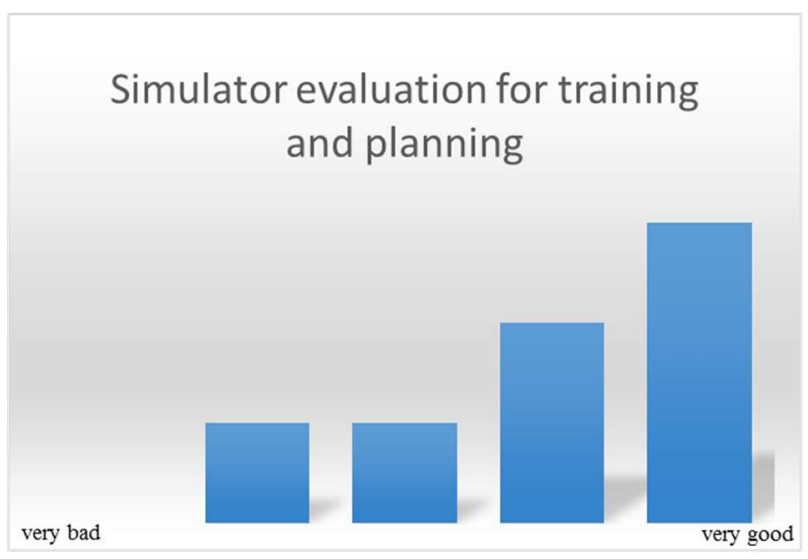

Figure 9. Simulator applicability for training and planning.

Comparing the real and the simulated data, some artefacts have been noticed: despite the point cloud being realistic, as well as noise and material effects have been simulated, some materials (e.g. glass) resulted a quite dense point cloud, which differs from reality (s. Fig.10). 


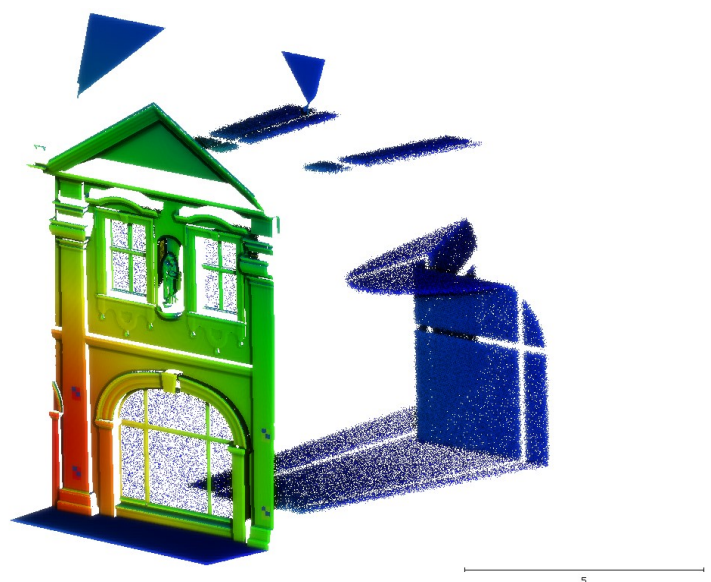

Figure 10. Glass material behaviour in simulator.

This clearly demonstrates that reflection and glass propagation functions are quite complex, and algorithms should be researched more deeply and improved.

\section{CONCLUSIONS AND OUTLOOK}

It can be concluded that VRscan3D:

- gives a useful 3D laser scanning visualisation for a hands-on process explanation of how to use a TLS device;

- $\quad$ is easy to operate and offers all modern performance of game environments;

- helps the user to learn basic terrestrial laser scanning parameters and interface usage and is useful for planning of an individual workflow;

- allows the generation of simulated 3D scan data comparable with real data, including noise, intensity, panoramic images and reflections.

- replaces real instruments for practical training if there is no access to real (expensive) equipment;

- $\quad$ is a useful tool for distance learning courses, e-learning and online teaching.

VRscan3D is available as a free demo version providing a generic scanner model and a limited number of scanning stations. A full version with different scanner models and unlimited functionality will be available in due time (see http://vrscan3d.com).

Furthermore, it is planned to integrate methods of serious gaming elements, i.e. active learning within a virtual environment, and to further develop them in an application-oriented manner.

\section{ACKNOWLEDGEMENTS}

The project is funded under the DAAD program for support for the internationalisation of Ukrainian higher education institutions - shaping the digital future together: German- Ukrainian higher education institution collaborations. The financial support is gratefully acknowledged. Further information is available on the project website:

https://iapg.jade-hs.de/projekte/virscan3d and software website: http://vrscan3d.com

\section{REFERENCES}

Chizhova, M., Popovas, D., Gorkovchuk, D., Gorkovchuk, J., Hess, M., Luhmann, T., 2020: Virtual terrestrial laser scanner simulator for digitalization of teaching environment: Concept and first results. Int. Arch. Photogramm. Remote Sens. Spatial Inf. Sci., XLIII-B5-2020. https://doi.org/10.5194/isprs-archivesXLIII-B5-2020-91-2020

Chizhova, M., Gorkovchuk, D., Kachkovskaya, T., Popovas, D., Gorkovchuk, J., Luhmann, T., Hess, M., 2021: Qualitative testing of an advanced terrestrial laser scanner simulator: users experience and feedback. Int. Arch. Photogramm. Remote Sens. Spatial Inf. Sci., 43, 29-35. https://doi.org/10.5194/isprsarchives-XLIII-B5-2021-29-2021

Dib, H., Adamo-Villani, N., \& Garver, S., 2014: Advances in engineering education An Interactive Virtual Environment for Learning Differential Leveling: Development and initial Findings. Advances in Engineering Education, 4(1), 1-17.

DiKule (2022): DiKuLe: Digitale Kulturen der Lehre entwickeln. https://www.uni-bamberg.de/dikule/

Falloon, G., 2019: Using simulations to teach young students science concepts: An Experiential Learning theoretical analysis. Computers and Education, 135, 138-159. https://doi.org/10.1016/j.compedu.2019.03.001

Kersten, T. P., Edler, D., 2020: Special Issue "Methods and Applications of Virtual and Augmented Reality in GeoInformation Sciences." PFG - Journal of Photogrammetry, Remote Sensing and Geoinformation Science, 88(2), 119-120. https://doi.org/10.1007/s41064-020-00109-w

Kuo, H.-L., Kang, S.-C., Lu, C.-C., Hsieh, S.-H., Lin, Y.-H., 2011: Using Virtual Instruments to Teach Surveying Courses: Application and Assessment. Computer Applications in Engineering Education, 19, 411-420.

Popovas, D., Chizhova, M., Gorkovchuk, D., Gorkovchuk, J., Hess, M., Luhmann, T., 2021: Teaching terrestrial laser scanning in cultural heritage using a virtual simulator. Proceedings of the joint international event 9th ARQUEOLÓGICA $2.0 \& 3 \mathrm{rd}$ GEORES, Valencia (Spain) https://doi.org/10.4995/Arqueologica9.2021.12091

Smart Didaktik (2022): M2: Innovative Methoden für eine smarte digitale Didaktik. https://www.unibamberg.de/dikule/teilprojekte/smarte-didaktik/

Vlachopoulos, D., Makri, A., 2017: The effect of games and simulations on higher education: a systematic literature review. International Journal of Educational Technology in Higher Education, 14(1). https://doi.org/10.1186/s41239-017-0062-1 